Int. J. Odontostomat., 8(1):47-52, 2014.

\title{
Localización de OPG en la Pulpa Dental del Ratón Albino Suizo
}

\author{
OPG Location in Dental Pulp of Albino Swiss Mice
}

\author{
Mario Alejandro Ortiz Salazar \& Liliana Salazar Monsalve*
}

ORTIZ, S. M. A. \& SALAZAR, M. L. Localización de OPG en la pulpa dental del ratón albino suizo. Int. J. Odontostomat., 8(1):47-52, 2014.

RESUMEN: En condiciones fisiológicas, el órgano dentinopulpar no se encuentra asociado a procesos de remodelación y resorción. La Osteoprotegerina (OPG) es una molécula del Factor de Necrosis Tumoral (TNF) que participa en la diferenciación y activación de osteoclastos, por lo que su papel en el control del proceso de reabsorción radicular interna debe ser determinado. Se tomaron secciones histológicas de $5 \mu \mathrm{m}$ tomadas de primeros molares de 8 hemimandíbulas de ratón y fueron sometidas a proceso de inmunohistoquímica con la técnica de complejo avidina-biotina peroxidasa para la detección de OPG. Se identificó la presencia de OPG en las regiones central y periférica de la pulpa de ratones de 4 y 12 semanas. La pulpa de ratones albino suizo mostró presencia diferencial de OPG en las zonas central y periférica.

PALABRAS CLAVE: diente, osteoprotegerina, reabsorción.

\section{INTRODUCCIÓN}

El metabolismo del tejido óseo y demás tejidos mineralizados se encuentra bajo el control de numerosos factores de naturaleza hormonal, autocrina, local y transcripcional, los cuales afectan la actividad de los diferentes tipos de células implicadas en el mantenimiento de la masa tisular (Raisz, 1999). En el tejido óseo estas células se integran en el proceso de remodelación a través de las Unidades Multicelulares Oseas (BMU) que involucran osteoblastos, osteocitos y osteoclastos (Lerner, 2004).

Investigaciones recientes han hecho evidente que los osteoblastos, además de otras células del tejido conectivo como Linfocitos T y Odontoblastos, están implicados en el control del proceso de actividad y diferenciación de los osteoclastos a través de contacto célula a célula con los progenitores mononucleares del linaje monocito-macrófago (Sakata et al., 1999; Rani \& MacDougall, 2000; Kuntz et al., 2001). En los últimos años se han encontrado las moléculas responsables de este control, las cuales pertenecen a la superfamilia del Factor de Necrosis Tumoral (TNF). De esta manera, la expresión del Ligando del Receptor Activador Nuclear Kappa B (RANKL) en la superficie de los osteoblastos y la activación de su receptor RANK en los precursores mononucleares (preosteoclastos) se convierte en el requisito para la formación de osteoclastos (Boyle et al., 2003). Esta unión puede ser bloqueada por la proteína Osteoprotegerina (OPG), otro miembro de la superfamilia TNF, secretada por los mismos osteoblastos. Esta proteína, en conjunto con RANK y RANKL, conforman el eje del control del metabolismo óseo (Aubin \& Bonnelye, 2000; Feige, 2001).

En condiciones fisiológicas, los tejidos dentales no sufren procesos de resorción, a excepción del proceso de resorción radicular que es llevado a cabo durante la exfoliación de la dentición temporal. Sin embargo, durante el establecimiento de procesos patológicos de los tejidos dentales, es posible identificar la activación de procesos resortivos que afectan al hue-

Odontólogo, Magister en Ciencias Básicas Médicas. Especialista en Ortodoncia. Profesor Auxiliar, Departamento de Morfología, Grupo de Tejidos Blandos y Mineralizados, Universidad del Valle, Cali, Colombia.

* Fisioterapeuta, Maestría en Morfología. Profesor Asociado, Departamento de Morfología, Universidad del Valle, Cali, Colombia. Trabajo de Grado para optar por el titulo Magister en Ciencias Básicas Medicas, Universidad del Valle, Cali, Colombia.

Proyecto financiado por Vicerrectoría de Investigaciones, Universidad del Valle, Cali, Colombia. 
so alveolar, cemento y/o dentina (Fuss et al., 2003). Estas células se encuentran bajo el control del eje RANK/RANKL/OPG (Sakata et al.; Rani \& MacDougall). Por lo tanto, el propósito del presente estudio fue determinar a través de inmunohistoquímica la presencia de Osteoprotegerina en la pulpa de ratones albinos suizos de 4 y 12 semanas de edad.

\section{MATERIAL Y MÉTODO}

Se utilizaron ratones machos de la cepa Albino Suizo de 4 y 12 semanas (4 de cada uno) provenientes del Bioterio de la Facultad de Salud de la Universidad del Valle. Todos los procedimientos fueron aprobados por el Comité de ética en Investigación Animal de la Facultad de Salud. A la edad de evaluación (4 y 12 semanas respectivamente) los ratones fueron sometidos a eutanasia intraperitoneal con Pentobarbital sódico, seguido de perfusión cardiaca con Paraformaldehido al $4 \%$. Las muestras obtenidas consistieron en 8 hemimandíbulas sometidas a fijación por inmersión en paraformaldehido al $4 \%$ a $24^{\circ} \mathrm{C}$ durante 48 horas. Posteriormente fueron lavadas con solución fisiológica a pH 7,4 durante una hora y sometidas a protocolo de decalcificación con EDTA al $5 \%$ durante 10 días a $4^{\circ} \mathrm{C}$. Las muestras se sometieron a deshidratación en gradiente ascendente de alcoholes, aclaramiento en Xilol e inclusión en Paraplast $₫$, para luego ser cortadas en sentido mesio-distal en un micrótomo hasta obtener muestras de $5 \mu \mathrm{m}$ de grosor.

Inmunotinción. Las muestras fueron sometidas a desparafinización con xilol y metanol, para posteriormente ser sometidas a rescate antigénico con solución de EDTA a $18 \%$ a $125^{\circ} \mathrm{C}$ durante 15 minutos (Imam et al., 1995). Las placas se enfriaron durante 30 minutos en agua corriente y se colocaron otros 30 minutos en PBS. Luego se colocaron en cámara humidificada y se incubaron peróxido de Hidrógeno al $1 \%$ para bloquear la peroxidasa endógena, se lavaron en PBS con Triton $X$ y se incubaron con suero Bloqueador al $1,5 \%$. Las placas fueron incubadas con el Anticuerpo Primario (Goat IgG Anti-OPG) (Santa Cruz Biotechnology OPG N-20: sc-8468) en concentración 1:100 por una noche a $4^{\circ} \mathrm{C}$. Al día siguiente las muestras se lavaron con PBS con Triton y fueron incubadas en el Anticuerpo Secundario Biotinilado durante una hora (Santa Cruz Biotechnology ABC Staining System sc-2023). Luego fueron lavadas con PBS e incubadas en el reactivo AB (Avidina - Biotina) durante treinta minutos, se reveló en sustrato de Peroxidasa - Diaminobenzidina (DAB substrate, Dako DIN) y se contrastaron con Hematoxilina Férrica. Para su evaluación se montaron en medio permanente con cubreobjetos (Permount, Fisher Scientific, EE.UU.). Como control positivo para osteoprogeterina se utilizaron muestras de cáncer de mama provenientes del archivo del Laboratorio de Patología de la Universidad del Valle (Fisher et al., 2006; Park et al., 2003). Los controles inmunohistoquímicos se realizaron por: 1) Reemplazo del anticuerpo primario por PBS y 2) Omisión de anticuerpo secundario goat lgG. Las secciones sometidas a este protocolo no presentaron inmunotinción.

Evaluación. Las muestras fueron evaluadas bajo microscopio de luz utilizando un Microscopio Óptico Olympus adaptado a una cámara CANON Power Shot A80 con adaptador CANON LA-DC52D. La respuesta de las muestras sometidas a Protocolo de Inmunohistoquímica para detectar la presencia de OPG se evaluó con los siguientes parámetros: Negativa (-), Leve (+), Moderada (++) y Marcada (+++), de acuerdo con la intensidad de tinción del cromógeno, considerándose negativa la ausencia total de tinción de cromógeno y como Marcada la presencia intensa de coloración café. El análisis de la inmunoreactividad se determino dividiendo la pulpa en dos regiones según Pinzón et al. (1967) para mamíferos inferiores: Región central de la pulpa y Región periférica de la pulpa.

\section{RESULTADOS}

Presencia de OPG en ratones de cuatro semanas. La Presencia de OPG a nivel de la pulpa dental, evaluada a través de inmunohistoquímica, presenta variaciones dependiendo de la edad y de la ubicación en la región periférica y central. En la región periférica se observa una expresión francamente positiva (OPG +++) a nivel de los odontoblastos con expresión a nivel del citoplasma. En estas células no se identifica reactividad a nivel nuclear y la reactividad en membrana celular no es clara. En la región central de la pulpa se observa expresión moderada (OPG++) en los fibroblastos pulpares, la cual se ubica a nivel del citoplasma. También es posible identificar expresión leve (OPG+) a nivel de la matriz extracelular y en las paredes de los vasos sanguíneos. En esta última, corresponde a la localización de las células endoteliales. 
Presencia de OPG en ratones de doce semanas. La presencia de OPG a nivel de la pulpa dental presenta variaciones dependiendo de la edad y de la ubicación en la región periférica y central. En la región periférica se observa una disminución en la expresión de OPG con respecto a la identificada en ratones de 4 semanas. La reactividad es catalogada como leve $(\mathrm{OPG}+)$ a nivel del citoplasma de los odontoblastos con una distribución selectiva, con énfasis en la presencia de la proteína en los extremos proximal y distal de la célula. Se nota una acumulación de Osteoprotegerina en la proximidad a la predentina. En

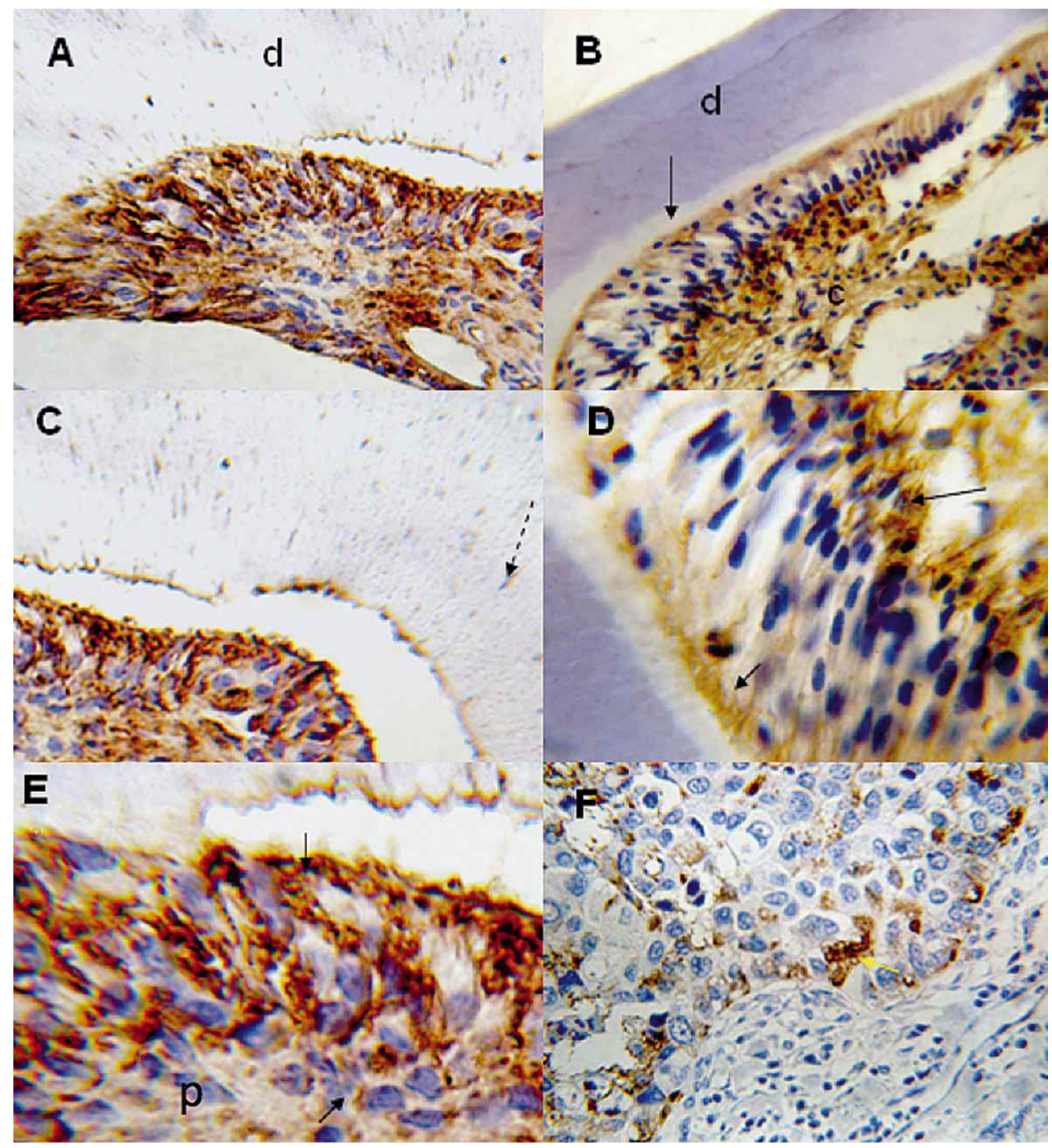

Fig. 1. Microfotografías de Cortes Histológicos de Molares después de Reacción inmunohistoquímica para OPG. A, C y E corresponden a ratones de 4 semanas. B y D corresponden a ratones de 12 semanas. F Corresponde a control positivo de Carcinoma de Mama. En A y $C$ se puede observar la intensa reacción inmunohistoquímica en la región central y periférica de la pulpa (Flecha continua). Nótese la reacción negativa en la matriz de la dentina calcificada (d) y la poca reacción en algunos túbulos dentinarios (Flecha discontínua). En E se observa una magnificación de la región periférica de la pulpa $(p)$, en especial la reacción positiva severa en la región de contacto dentinal. En B se observa la expresión en ratones de 12 semanas en la región central y periférica. Nótese la ausencia de reacción en la dentina (d) y en la predentina (flecha continua). La magnificación en D permite observar expresión positiva moderada de OPG en el cuerpo de los odontoblastos y la reacción positiva severa en la zona subodontoblástica (Ver flecha continua). $\mathrm{F}$ presenta los hallazgos del control positivo en Cáncer de Mama. Aumento original de 10X en A, B y F, 40X en C y $100 \mathrm{X}$ en D y E. 
la región subodontoblástica ubicada justo por debajo de los odontoblastos, se identifica una expresión moderada de OPG (OPG++), aunque en algunas células es severa. La expresión de la proteína sigue el contorno citoplasmático celular y se observa la expresión de proyecciones celulares. En esta región se encuentran las células dendríticas y las células madre mesenquimatosas. En la región central de la pulpa se identifica una expresión marcada (OPG +++) distribuida a nivel del citoplasma de los fibroblastos pulpares y especialmente a nivel de la matriz extracelular.

Los resultados de los grupos estudiados pueden ser observados en la Tabla I y en la Figura 1A, C y $\mathrm{E}$ para ratones de 4 semanas; $1 \mathrm{~B}$ y $\mathrm{D}$ para ratones de 12 semanas.

Tabla I. Perfil de detección osteoprotegerina en órgano dentinopulpar de ratones de 4 y 12 Semanas.

\begin{tabular}{llcc}
\hline & & OPG 4 semanas & OPG 12 semanas \\
\cline { 2 - 3 } Matriz de dentina & Dentina & - & - \\
& Predentina & + & - \\
& Prolongación odontoblástica & + & - \\
\multirow{2}{*}{ Pulpa dental } & Región periférica & +++ & ++ \\
& Región central & ++ & +++ \\
\hline
\end{tabular}

(-) Ausente; (+) Leve; (++) Moderado; (+++) Marcado.

\section{DISCUSIÓN}

Se ha propuesto que la Osteoprotegerina (OPG) puede ser la clave en el control del metabolismo óseo y mineral (Khosla, 2001), ya que su actividad biológica está centrada en inhibir la diferenciación de Osteoclastos y dificultar su adhesión a las superficies (Yasuda et al., 1998). De esta manera participaría en la inhibición de los procesos resortivos (Nakamura et al., 2002).

El presente trabajo describió de manera cualitativa la expresión de Osteoprotegerina en el Órgano Dentino Pulpar del Ratón Albino Suizo. Los estudios in vivo de la expresión de OPG en los tejidos dentales incluyendo la pulpa dental son escasos en la literatura científica; de acuerdo a la revisión bibliográfica realizada, este es el segundo estudio que evalúa la expresión de OPG en pulpa dental in vivo y el primero en realizarlo en ratones. En este estudio se encontró que sin importar la edad del ratón, se observaba una presencia marcada (+++) de OPG por parte de las células correspondientes a la Región periférica de la pulpa, descritas como odontoblastos en el estudio histoquímico. Estos hallazgos se encuentran en concordancia con los estudios in vitro presentados por Rani \& MacDougall y Sakata et al., quienes describieron la habilidad de expresar mRNA de OPG por parte de las células dentales de origen mesenquimal y no por parte de las epiteliales. Uchiyama et al. (2009), encontraron que la expresión de OPG yRANKL por parte de las células pulpares y del ligamento periodontal per- mite soportar la diferenciación y mantenimiento de osteoclastos in vitro.

La presencia de OPG en estudios in vitro es concordante con los resultados in vivo presentados por Kuntz et al., quienes al investigar la presencia de OPG en pulpas dentales humanas sanas e inflamadas encontraron alta reactividad en la región periférica de la pulpa $\sin$ importar el estado de salud de las mismas.

Con respecto a la expresión de OPG en la región central de la pulpa, se encontró una reacción intensa (+++) en ratones de 4 semanas y moderada $(++)$ en ratones de 12 semanas. Esta expresión se asocia a células con características morfológicas similares a fibroblastos pulpares. Estos hallazgos inmunohistoquímicos se encuentran en contraposición con los reportados por Kuntz et al., quienes observaron una expresión leve en las regiones centrales de las pulpas humanas sanas. Estas diferencias podrían deberse a razones tales como el tipo de pulpa estudiada y la metodología utilizada en cada experimento.

En general, los estudios in vivo e in vitro de la expresión de OPG en el órgano dentinopulpar, muestran que no existe claridad sobre el papel que juega este factor en la pulpa dental. Si bien los estudios in vitro muestran que OPG actúa como inhibidor de Osteoclastos (Sakata et al.; Rani \& MacDougall), la 
actividad de OPG como supresor de la osteoclastogénesis y de los osteoclastos en los tejidos dentales mineralizados, debe ser estudiada con mayor profundidad.

Desde esta perspectiva el proceso de resorción radicular fisiológico es llevado a cabo por la integración de las células de los tejidos óseos y dentales que permiten el control molecular para la aparición de los osteoclastos (Harokopakis-Hajishengallis, 2007). Con estos conceptos, Helfrich (2005) ha revisado las diferentes patologías que alteran el desarrollo de los osteoclastos y sus efectos sobre los tejidos dentales, encontrando alteraciones en la erupción dental y odontomas en osteoporosis recesiva y pérdida dental precoz en Enfermedad de Piaget.

La actividad de OPG ha sido descrita en los procesos de desarrollo de la periodontitis (Liu et al., 2003). La proporción entre los niveles de OPG y RANKL medidos en fluido crevicular parece ser un adecuado marcador biológico del proceso de osteolisis asociado a la periodontitis (Bostanci et al., 2007).
El estudio del roll de la OPG en tejidos dentales puede abrir una puerta al conocimiento del mecanismo por medio del cual los tejidos dentales sufren remodelación.

\section{CONCLUSIONES}

La Osteoprotegerina se encontró expresada a nivel del Órgano Dentinopulpar, siendo alta su presencia a nivel de la pulpa dental y leve a nivel de la matriz de dentina.

Existen diferencias en la presencia de Osteoprotegerina a nivel de la pulpa asociada a la edad. Los niveles de reactividad a OPG en pulpa dental fueron más elevados en ratones de 4 semanas en comparación con los de 12 semanas.

Se asoció un patrón de expresión de Osteoprotegerina con la organización histotopográficas de la pulpa dental del ratón.

ORTIZ, S. M. A. \& SALAZAR, M. L. OPG location in dental pulp of albino Swiss mice. Int. J. Odontostomat., 8(1):47-52, 2014.

ABSTRACT: Under physiological conditions, the dentinopulpar complex is not associated with remodeling and resorption. Osteoprotegerin (OPG) is a TNF molecule associated with osteoclast activation and differentiation. Therefore, OPG role in internal root resorption must be determined. We obtained histological sections of $5 \mu \mathrm{m}$ in first molars from 8 hemimandibles of mice, which were subjected to the avidin-biotin peroxidase complex immunohistochemical procedure for detection of OPG. Differential OPG presence was identified in central and peripheral pulp in dental pulp of 4 and 12 week-old mice.

KEY WORDS: tooth, osteoprotegerin, resorption.

\section{REFERENCIAS BIBLIOGRÁFICAS}

Aubin, J. E. \& Bonnelye, E. Osteoprotegerin and its ligand: A new paradigm of Regulation of Osteoclastogenesis and bone resorption. Osteoporos. Int., 11(11):905-13, 2000.

Bostanci, N.; I'Igenli, T.; Emingil, G.; Afacan, B.; Han, B.; Töz, H.; Atilla, G.; Hughes, F. J. \& Belibasakis, G. N. Gingival crevicular fluid levels of RANKL and OPG in periodontal diseases: implications of their relative ratio. J. Clin. Periodontol., 34(5):370-6, 2007.

Boyle, W. J.; Simonet, W. S. \& Lacey, D. L. Osteoclast differentiation and activation. Nature, 423(6937):33742, 2003.

Feige, U. Osteoprotegerin. Ann. Rheum. Dis., 60(Suppl. 3):iii81-4, 2001.
Fisher, J. L.; Thomas-Mudge, R. J.; Elliott, J.; Hards, D. K.; Sims, N. A.; Slavin, J.; Martin, T. J. \& Gillespie, M. T. Osteoprotegerin Overexpression by Breast Cancer Cells Enhances Orthotopic and Osseous Tumor Growth and Contrasts with That Delivered Therapeutically. Cancer Res., 66(7):3620-8, 2006.

Fuss, Z.; Tsesis, I. \& Lin, S. Root Resorption - Diagnosis, Classification and treatment choices based on stimulation factors. Dent. Traumatol., 19(4):175-82, 2003.

Harokopakis-Hajishengallis, E. Physiologic root resorption in primary teeth: molecular and histological events. J. Oral Sci., 49(1):1-12, 2007.

Helfrich, M. Osteoclast diseases and dental abnormalities. Arch. Oral Biol., 50(2):115-22, 2005. 
Imam, S. A.; Young, L.; Chaiwun, B. \& Taylor, C. R. Comparison of two microwave based antigen-retrieval solutions in unmasking epitopes in formalin-fixed tissue for immunostaining. Anticancer Res., 15(4):1153-8, 1995.

Khosla, S. Minireview: The OPG/RANKL/RANK System. Endocrinology, 142(12):5050-5, 2001.

Kuntz, K. A.; Brown, C. E. Jr.; Legan, J. J. \& Kafrawy, A. H. An Immunhistochemical Study of Osteoprotegerin in the Human Dental Pulp. J. Endod., 27(11):666-9, 2001.

Lerner, U. H. New Molecules in the Tumor Necrosis factor Ligand and Receptor Superfamilies with importance for Physiological and Pathological Bone Resorption. Crit. Rev. Oral Biol. Med., 15(2):64-81, 2004.

Liu, D.; Xu, J. K.; Figliomeni, L.; Huang, L.; Pavlos, N. J.; Rogers, M.; Tan, A.; Price, P. \& Zheng, M. H. Expression of RANKL and OPG mRNA in periodontal disease: Possible involvement in bone destruction. Int. J. Mol. Med., 11(1):17-21, 2003.

Nakamura, H.; Tsuji, T.; Hirata, A. \& Yamamoto, T. Localization of Osteoprotegerin (OPG) on bone surfaces and cement lines in rat tibia. J. Histochem. Cytochem., 50(7):94553, 2002.

Park, H. R.; Min, S. K.; Cho, H. D.; Kim, D. H.; Shin, H. S. \& Park, Y. E. Expression of osteoprotegerin and RANK ligand in breast Cancer bone metastasis. J. Korean Med. Sci., 18(4):541-6, 2003.

Pinzón, R. D.; Kozlov, M. \& Burch, W. P. Histology of Rat Molar Pulp at different ages. J. Dental Res., 46(1):2028, 1967.

Raisz, L. G. Physiology and Pathophysiology of bone remodeling. Clin. Chem., 45(8 Pt. 2):1353-8, 1999.

Rani, C. S. \& MacDougall, M. Dental cells express factors that regulate bone resorption. Mol. Cell. Biol. Res. Commum., 3(3):145-52, 2000.

Sakata, M.; Shiba, H.; Komatsuzawa, H.; Fujita, T.; Ohta, K.; SuGai, M.; Suginaka, H. \& Kurihara, H. Expression of osteoportegerin (osteoclastogenesis inhibitory factor) in cultures of human dental mesenchymal cells and epithelial cells. J. Bone Miner. Res., 14(9):1486-92, 1999.

Uchiyama, M.; Nakamichi, Y.; Nakamura, M.; Kinugawa, S.; Yamada, H.; Udagawa, N. \& Miyazawa, H. Dental Pulp and Periodontal Ligament Cells Support Osteoclastic Differentiation. J. Dent. Res., 88(7):609-14, 2009.

Yasuda, H.; Shima, N.; Nakagawa, N.; Mochizuki, S.; Yano, K.; Fujise, N.; Sato, Y.; Goto, M.; Yamaguchi, K.; Kuriyama, M.; Kanno, T.; Murakami, A.; Tsuda, E.; Morinaga, T. \& Higashio, K. Identity of
Osteoclastogenesis Inhibitory factor (OCIF) and Osteoprotegerin (OPG): A mechanism by which OPG/ $\mathrm{OCIF}$ inhibits osteoclastogenesis in vitro. Endocrinology, 139(3):1329-37, 1998.

Dirección para Correspondencia:

Mario Alejandro Ortiz

Departamento de Morfología

Universidad del Valle

Sede San Fernando

Calle 4B No 36-00 Edif. 116

Cali

COLOMBIA

Email: mariortiz@yahoo.com

Recibido : 25-07-2013

Aceptado: 31-01-2014 